\title{
Correction to: DIIS Theory and Methodology in Think Tanks
}

\section{Correction to:}

\section{J. Pan, DIIS Theory and Methodology in Think Tanks, https://doi.org/10.1007/978-981-16-0618-2}

In the original version of the book, belated corrections as regards author names and affiliations have been received in Frontmatter, Backmatter and in Chapters. The book has been updated with the changes.

The updated versions of these chapters can be found at https://doi.org/10.1007/978-981-16-0618-2_1, https://doi.org/10.1007/978-981-16-0618-2_2, https://doi.org/10.1007/978-981-16-0618-2_3, https://doi.org/10.1007/978-981-16-0618-2_4, https://doi.org/10.1007/978-981-16-0618-2 